\title{
ORIGINAL ARTICLE Retrospective study for risk factors for febrile UTI in spinal cord injury patients with routine concomitant intermittent catheterization in outpatient settings
}

\author{
S Mukai ${ }^{1}$, K Shigemura ${ }^{2,3}, \mathrm{M} \mathrm{Nomi}^{1}$, A Sengoku${ }^{1}, \mathrm{~F}$ Yamamichi $^{2}$, M Fujisawa ${ }^{2}$ and S Arakawa ${ }^{2,3}$
}

Study design: Retrospective study.

Objectives: The objective of this study was to investigate the clinical risk factors for febrile urinary tract infection (UTI) in spinal cord injury-associated neurogenic bladder (NB) patients who perform routine clean intermittent catheterization (CIC).

Setting: Rehabilitation Hospital, Kobe, Japan.

Methods: Over a 3-year period, we retrospectively assessed the clinical risk factors for febrile UTI in 259 spinal cord injury patients diagnosed as NB and performing routine $\mathrm{CIC}$ with regard to the factors such as gender, the presence of pyuria and bacteriuria, and the categories of the American Spinal Injury Association (ASIA) impairment scale.

Results: A total of 67 patients had febrile UTI in the follow-up period, with 57 cases of pyelonephritis, 11 cases of epididymitis and 2 cases of prostatitis, including the patients with plural infectious diseases. The causative bacteria were ranked as follows: Escherichia coli (74 cases), Pseudomonas aeruginosa (17 cases), Enterococcus faecalis (14 cases) and Klebsiella pneumoniae (12 cases). Antibiotic-resistant E. coli were seen, with $10.5 \%$ instances of extended-spectrum $\beta$-lactamase (ESBL) production and $23.8 \%$ of fluoroquinolone resistance. Multivariate analyses of clinical risk factors for febrile UTI showed that gender (male, $P=0.0431$ ), and ASIA impairment scale $C$ or more severe $(P=0.0266)$ were significantly associated with febrile UTI occurrence in NB patients with routine $\mathrm{CIC}$.

Conclusion: Our data demonstrated gender (male) and ASIA impairment scale C or more severe were significantly associated with febrile UTI occurrence in NB patients using routine CIC. Further prospective studies are necessary to define the full spectrum of possible risk factors for febrile UTI in these patients.

Spinal Cord (2016) 54, 69-72; doi:10.1038/sc.2015.170; published online 13 October 2015

\section{INTRODUCTION}

Neurogenic bladder (NB) is a voiding disturbance caused by neurogenic diseases. Cerebrovascular disease and spinal cord dysfunction, due to, for instance, spinal cord injury, and neurodegenerative disease are representative causes of NB. The main symptoms are voiding and storage disturbance, and urinary tract stones and urinary tract infection (UTI) are often involved. One of main therapeutic strategies is urinary tract catheterization, but long duration of urethral catheter storage can cause complications such as urogenital infection, bladder stone, urethral injury or urinary tract fistula. ${ }^{1}$ Substituting intermittent catheterization may decrease such complications.

Clean intermittent catheterization (CIC) is considered as the first choice for the patients whose inability to void effectively causes overextension of the bladder wall and increase of intravesical pressure leading to the risk of UTI, vesicoureteral reflux (VUR) and upper urinary tract dysfunction. CIC is recommended for patients with lower urinary tract symptoms (LUTS) and uncontrollable autonomic hyperreflexia. The 2005 'Clinical Practice Guideline For Lower Urinary Tract Dysfunction In Patients With Chronic Spinal Cord Injury' from the Japanese Continence Society recommends CIC for the patients who have more than $100 \mathrm{ml}$ of residual urine, findings of bladder deformation or VUR on voiding cystography, or detrusor-sphincter dyssynergia (DSD), or bladder compliance $<20 \mathrm{ml}$ per $\mathrm{H}_{2} \mathrm{O}$. ${ }^{2}$

CIC tends to cause fewer urinary tract complications compared with urethral indwelling catheterizations, and the rate of febrile UTI occurrence with indwelling catheters has been reported as once/3.9 years, ${ }^{3}$ whereas patients on CIC of three times or more per day reportedly had febrile UTI of once/10 years. ${ }^{4}$

There are several reports regarding the risk factors for febrile UTI in NB patients, especially focusing on spinal cord injury. A study of 140 spinal cord injury patients excluding indwelled urethral catheter patients found that $75 \%$ of quadriplegic patients and $92 \%$ of patients with complete injury had hydronephrosis, and $86 \%$ of quadriplegic patients and $100 \%$ of the patients with complete injury had VUR. ${ }^{5}$ Quadriplegia and paralysis are considered as greater risk factors for upper urinary tract dysfunction compared with paraplegia and paresis.

Neurological examination of the sacral region related to urinary tract dysfunction appears in the first category of the American Spinal Injury Association (ASIA) evaluation sheet. ${ }^{6}$ The preservation of sensory function in S4-S5 and the ability to voluntarily contract the

\footnotetext{
${ }^{1}$ Department of Infection Control and Prevention, Hyogo Prefectural Rehabilitation Center, Kobe, Japan; ${ }^{2}$ Department of Surgery, Division of Urology, Kobe University Graduate School of Medicine, Kobe, Japan and ${ }^{3}$ Department of Infection Control and Prevention, Kobe University Hospital, Kobe, Japan

Correspondence: Dr K Shigemura, Department of Urology, Kobe University Graduate School of Medicine, 7-5-1 Kusunoki-Cho, Chuo-Ku, Kobe 650-0017, Hyogo, Japan.

E-mail: yutoshunta@hotmail.co.jp

Received 22 April 2015; revised 11 August 2015; accepted 1 September 2015; published online 13 October 2015
} 
levator ani muscle are important for discriminating complete and incomplete injury. Complete versus incomplete injury and the ability to contract the levator ani muscle are related to $\mathrm{DSD}^{7}$ and therefore the severity of spinal cord diseases is considered to correlate with the risk of febrile UTI. As UTI remains one of the main complications in these patients, we investigated the clinical risk factors for febrile UTI in NB patients using CIC.

\section{MATERIALS AND METHODS}

\section{Patients}

The data were gathered from 259 spinal cord injury-related NB patients in outpatient settings without any ambulatory patients, and trauma was the main cause (Table 1) who required CIC from June 2011 to June 2014 in the Department of Urology of Hyogo Prefectural Rehabilitation Central Hospital. This retrospective study was follow patients up 3 years. All patients consented to the study in writing. The diagnosis of NB was based on voiding disturbances caused by an underlying disease such as spinal cord diseases with abnormal findings on urodynamic study (UDS), such as detrusor overactivity, DSD, lowcompliance bladder in cervical or thoracic spinal cord diseases patients and overextended bladder or low detrusor activity. The study was performed in accordance with the principles of the Declaration of Helsinki.

\section{Antibiotic susceptibilities}

Susceptibility testing was performed according to the Clinical and Laboratory Standards Institute criteria. Extended-spectrum $\beta$ - lactamase (ESBL) production tests were performed as in our previous study. ${ }^{8}$ Resistant strains in this study category were defined as ESBL-producing bacteria, methicillin-resistant Staphylococcus aureus (MRSA) and multidrug-resistant Pseudomonas aeruginosa

Table 1 Patient demographics

\begin{tabular}{lc}
\hline$n$ & 259 \\
Male & $220(84.9 \%)$ \\
Female & $39(15.1 \%)$ \\
Age (years): median (range) & $47(12-90)$ \\
& \\
Level of spinal cord injury & \\
Cervical & $86(33.2 \%)$ \\
Upper thoracic & $50(19.3 \%)$ \\
Lower thoracic & $88(34.0 \%)$ \\
Lumbar & $34(13.1 \%)$ \\
Sacral & $1(0.4 \%)$ \\
& \\
Underlying diseases & \\
Traumatic & \\
Trauma & \\
Nontraumatic & $207(79.9 \%)$ \\
Spinal infarction & \\
Cerebral A-V malformation & $10(3.9 \%)$ \\
Spinal tumor & $8(3.1 \%)$ \\
Myelitis & $6(2.3 \%)$ \\
Acute disseminated encephalomyelitis & $4(1.5 \%)$ \\
Epidural hematoma & $4(1.5 \%)$ \\
Lumbar spinal canal stenosis & $3(1.2 \%)$ \\
Cervical spondylosis & $3(1.2 \%)$ \\
Pyogenic spondylitis & $2(0.8 \%)$ \\
Others & $2(0.8 \%)$ \\
Use of continuous catheterization at night & $10(3.9 \%)$ \\
Number of times of ClC per day: median (range) & $113(43.6 \%)$ \\
Anticholinergics & $7(1-20)$ \\
Anticholinergics+ $\beta-3$ adrenergic agonist & $193(74.5 \%)$ \\
Other bladder pressure decreasing treatment & $10(3.9 \%)$ \\
\hline & None \\
\hline
\end{tabular}

Abbreviation: CIC, clean intermittent catheterization.
(MDRP) defined as resistant to imipenem, ciprofloxacin and amikacin, or pre-MDRP was defined as resistant to two of them.

\section{Indication for CIC and diagnosis of febrile UTI}

The indications for CIC were as follows: (1) urinary retention, (2) repeated febrile UTI and (3) hydronephrosis due to bladder overflow. The diagnosis of febrile UTI was fever of $38^{\circ} \mathrm{C}$ or more, and urine white blood cell of 10 or more per high power field (hpf) with no other symptoms causing such a high fever. The patients were required to visit outpatients ward or emergency rooms in case of UTI or suspected UTI in addition to scheduled follow-up, and the diagnosis of UTI was performed at those visits. We included urogenital infection such as prostatitis and epididymitis in febrile UTI., ${ }^{9,10}$

\section{Investigated factors}

We tested the following potential risk factors for febrile UTI: (1) gender, (2) severity of spinal cord injury by ASIA impairment scale, (3) the duration of spinal cord injury or diseases, (4) pyuria (with 10 or more white blood cell: white blood cell counts per hpf) and bacteriuria: they were regarded as positive if they were seen in $50 \%$ or more frequency over all their office visits in 3 years study periods (We tested these urine tests every time of office visits.), (5) the number of CIC performance per day and (6) uses of anticholinergics and $\beta-3$ agonists.

\section{Statistical analyses}

Univariate and multivariate tests were performed using StatView 5.0 software (Abacus Concepts Inc., Berkley, CA, USA). Forward stepwise logistic regression analysis was conducted to determine the association between the various parameters mentioned above and febrile UTI. Statistical significance was established at the 0.05 level.

Igawa et al. ${ }^{1}$ recommended CIC of four to six times a day, and therefore we analyzed the number of CIC per day with four and six times as cutoff value and excluded the cases with nocturnal catheterization.

\section{RESULTS}

\section{Patient demographics and ASIA impairment scale}

The patient demographics are shown in Table 1. The ASIA impairment scale categorized 166 patients as A, 32 patients as B, 30 patients as $\mathrm{C}, 26$ patients as $\mathrm{D}$ and 5 patients as $\mathrm{E}$ (Table 1).

\section{Febrile UTI}

A total of $67(25.8 \%)$ patients had febrile UTI in the follow-up periods, with 57 cases of pyelonephritis, 11 cases of epididymitis and 2 cases of prostatitis, including the patients with plural infectious diseases. As to infectious events, 129 events of febrile UTI were observed, including 114 cases of pyelonephritis, 13 cases of epididymitis and 2 cases of prostatitis.

\section{Urine cultures}

A total of 88 UTI patients (including repeated UTIs) submitted urine samples. A total of 147 samples were submitted, of which 127 were bacterial-positive. A total of 181 bacteria were isolated. The causative bacteria were ranked as shown in the table, representatively, Escherichia coli (74 cases). In addition, antibiotic-resistant strains were seen in $29(16.0 \%)$ isolates, including ESBL producers: 21 (11.6\%). In addition, the rate of ESBL producers in all E. coli isolates was $28.4 \%$ and this accounted for $90.4 \%$ (19/21 strains) of all ESBL-producing isolates (Table 2).

\section{Pyuria and bacteriuria}

Pyuria was seen in $43.4 \%$ of all tested samples and $60.9 \%$ of the patients had bacteriuria. In terms of the association of pyuria and bacteriuria with the presence or absence of febrile UTI, for pyuria, 
43.3\% had febrile UTI and $43.9 \%$ did not $(P=0.895)$; for bacteriuria, $60.4 \%$ had febrile UTI and $62.1 \%$ did not $(P=0.837)$.

\section{The number of CIC per day}

We found that the median value of the number of CIC per day was seven times but there were no significant relationship between four times or less and six times or less of the number of CIC per day and the occurrence of UTI (Tables 1 and 3).

\section{Risk factors for febrile UTI}

Our clinical risk factors for febrile UTI based on multivariate analyses were shown in Table 3. Briefly, gender (male, $P=0.0431$ ) and ASIA impairment scale $\mathrm{C}$ or more severe $(P=0.0266)$ were significantly associated with febrile UTI occurrence in NB patients using routine CIC (Table 3).

\section{DISCUSSION}

Regarding UTI in NB patients caused by spinal cord injury or dysfunction, symptomatic UTI reportedly occurs in $22-45 \%,{ }^{11}$

\section{Table 2 Causative bacteria}

\begin{tabular}{lc}
\hline Escherichia coli & $74(40.9 \%)$ \\
Pseudomonas aeruginosa & $17(9.4 \%)$ \\
Enterococcus faecalis & $14(7.7 \%)$ \\
Klebsiella pneumoniae & $12(6.6 \%)$ \\
Citrobacter freundii & $8(4.4 \%)$ \\
Klebsiella oxytoca & $7(3.9 \%)$ \\
Serratia marcescens & $7(3.9 \%)$ \\
Citrobacter koseri & $6(3.3 \%)$ \\
ESBL-producing bacteria & $21(11.6 \%)$ \\
Escherichia coli & $19(10.5 \%)$ \\
MRSA & $7(3.9 \%)$ \\
Pre-MDRP & $1(0.6 \%)$ \\
\hline
\end{tabular}

Abbreviations: ESBL, extended-spectrum $\beta$-lactamase; MDRP, multidrug-resistant Pseudomonas aeruginosa; MRSA, methicillin-resistant Staphylococcus aureus; pre-MDRP, resistance to two of imipenem, ciprofloxacin and amikacin.

Table 3 Risk factors for febrile UTI comparable with our data of $25.8 \%$. As mentioned above, the rate of febrile UTI in patients on CIC performance of three or more times a day is reportedly once per 10 years, ${ }^{4}$ whereas our patients averaged once per 6.6 years. This difference may be because our cases tended to use broad-spectrum antibiotics for longer periods of UTI treatments regardless of guideline recommendation. ${ }^{12}$ We have given broad- spectrum antibiotics in our follow-up periods: for instance, fluoroquinolones: $49.4 \%$ and third-generation cephalosporines: $22.8 \%$ (data not shown). This factor should be reconsidered and unnecessary broad-spectrum antibiotics need to be prevented.

In our urine culture data, the most often isolated febrile UTIcausative bacteria were E. coli: $40.9 \%$ followed by P. aeruginosa: $9.4 \%$ and Enterococcus spp.: $7.7 \%$. Nicolle et al. ${ }^{13}$ found that in their CIC patients the most often isolated febrile UTI-causative bacteria were E. coli (35\%), followed by K. pneumonia (26\%), P. aeruginosa (23\%) and Proteus mirabilis $(16 \%)$. The major difference is the rate of K. pneumonia isolation and there is a different trend of the rate of ESBL production: for instance, ESBL-producing bacteria are reported in Japan at a rate of $1.3-6.7 \%$, similar to our data $(0 \%),{ }^{14,15}$ but a much higher rate in the United States $(34 \%)^{16}$ and Turkey (33\%). ${ }^{17}$ Regarding antibiotic-resistant bacteria, Chong et al. ${ }^{18}$ found that $14.3 \%$ of all E. coli isolates in UTI were ESBL producers, whereas other studies reported $21.0 \%$ in CAUTI and found indwelling urethral catheterization was a risk factor for ESBL production. ${ }^{17}$ Our data showed a higher rate of ESBL production in E. coli (28.4\%). This difference in ESBL production rate is partly because of our frequent use of broad-spectrum antibiotics such as thirdgeneration cephalosporines or fluoroquinolones as mentioned above. Then, other reports suggest restricting the use of third-generation cephalosporines may inhibit ESBL production or contamination, ${ }^{19,20}$ and then anti-microbial stewardship need to be performed because our uses of antibiotics were in most cases decided by each physicians and some cases cannot be explained as to the reason of the selection of those antibiotics from retrospective chart review as mentioned above.

\begin{tabular}{|c|c|c|c|c|c|c|}
\hline \multirow[t]{2}{*}{ Factors } & \multicolumn{3}{|c|}{ Univariate } & \multicolumn{3}{|c|}{ Multivariate } \\
\hline & $H R$ & $95 \% \mathrm{Cl}$ & $\mathrm{P}$-value & $H R$ & $95 \% \mathrm{Cl}$ & P-value \\
\hline Gender (male) & 3.669 & $1.235-10.744$ & 0.0177 & 3.078 & $1.035-9.154$ & 0.0431 \\
\hline Age & 1.008 & $0.989-1.027$ & 0.418 & & & \\
\hline Urological surgery & 0.74 & $0.200-2.735$ & 0.6512 & & & \\
\hline Pyuria & 0.863 & $0.293-2.546$ & 0.7894 & & & \\
\hline Bacteriuria & 1.037 & $0.590-1.823$ & 0.8994 & & & \\
\hline Nitrite & 1.339 & $0.725-2.698$ & 0.3169 & & & \\
\hline Antibiotic-resistant bacteria & 0.838 & $0.469-1.498$ & 0.5507 & & & \\
\hline Trauma & 1.451 & $0.698-3.015$ & 0.3186 & & & \\
\hline Immune compromization & 1.537 & $0.587-4.028$ & 0.3817 & & & \\
\hline Injury of cervical or upper thoracic & 1.263 & $0.544-2.932$ & 0.5867 & & & \\
\hline ASIA classification C & 0.997 & $0.994-0.999$ & 0.0117 & 0.997 & $0.994-1.000$ & 0.0266 \\
\hline Duration after trauma & 1.001 & $0.999-1.004$ & 0.3314 & & & \\
\hline Noctural catheterization & 1.734 & 0.996-3.022 & 0.0519 & & & \\
\hline Anticholinergics & 1.278 & $0.689-2.368$ & 0.4362 & & & \\
\hline$\beta-3$ Adrenergic agonist & 0.53 & $0.145-1.938$ & 0.337 & & & \\
\hline Number of $\mathrm{CIC}$ per day (four times or more) & 1.343 & $0.893-1.391$ & 0.9741 & & & \\
\hline Number of CIC per day (six times or more) & 1.317 & $0.472-3.676$ & 0.5933 & & & \\
\hline
\end{tabular}

Abbreviations: ASIA, American Spinal Injury Association; 95\% Cl, 95\% confident interval; HR, hazard ratio.

Bold represents statistical significance. 
CIC or urinary tract catheterized patients often had pyuria or bacteriuria. The standard for bacteriuria in CIC patients is $10^{2}$ or more colony-forming unit (CFU) per $\mathrm{ml}$ but that of pyuria in CIC patients is controversial and shows variation (urine white blood cell of 5 or more or of 10 or more per hpf). ${ }^{11,21}$ The relationship between urine findings and symptomatic UTI in spinal cord injury or dysfunction patients with CIC showing cloudy urine had the highest accuracy $(83.1 \%)$ and the second highest positive predictive value $(61.3 \%)$ and sensitivity $(65.5 \%),{ }^{22}$ suggesting it is realistic to initiate antibiotic treatments in cases with both pyuria- and UTI-related symptoms.

Our data showed a higher rate of febrile UTI in males compared with females. Males tend to have bladder neck occlusion anatomically with a concomitant risk of increased intravesical pressure. The further involvement of NB is considered to increase those risks. As to bladder motor function, ASIA impairment scale A, B and C classes include dysfunctions or malfunctions that tend to produce excessive high intravesical pressure or urinary incontinence. As to bladder sensory function, class A involves complete disability creating a situation where the patients do not notice excessive urine storage or urinary incontinence. Even though there are very few studies in this area, Jayawardena et al. ${ }^{23}$ reported class A is at risk for positive urine culture and bacteriuria. Our data showed ASIA class C or more severe significantly related with UTI occurrence and it can be interpreted as that class is more injured as to motor (muscle) function than class D so that it may lead to low bladder compliance and possibility of VUR.

One important mechanism causing UTI is high intravesical pressures resulting from distention of the bladder wall and to prevent this, Igawa et al. ${ }^{1}$ recommended $\mathrm{CIC}$ of four to six times a day as mentioned above. ${ }^{1}$ However, CIC can be inconvenient in daily life and proper CIC schedules may not be followed by all patients. Our data did not show any significant factors for UTI as to the number of CIC per day and could not suggest the proper number of CIC per day as to the prevention of UTIs; however, we had the nocturnal catheterized patients in a definitive percentage of patient population and it needs caution to direct comparison. Therefore, educating CIC patients on how best to decrease their risk of UTI is important and should be an ongoing mission, ${ }^{24}$ in that, for instance, many times of CIC with keeping clean technique leading to low rate of frequency of UTI occurrence. ${ }^{4,25,26}$

We did not show significant relationship between anticholinergics or other bladder pressure-decreasing-treatments and UTI occurrence, and it is considered the double treatments for low-compliance bladder by CIC and those drugs mutually operated and make analysis more complicated.

We would like to emphasize the limitations of this study. First, this study is retrospective in nature and our antibiotic use included preventive ones without any evidence so that we could not include them in our investigation. Second, the standards for febrile UTI are not generally well documented in this patient population. Third, we have no available data of risk evaluation for febrile UTI by UDS. Further prospective studies need to be performed for definitive conclusions.

In conclusion, our data demonstrated that male gender, and ASIA impairment scale $\mathrm{C}$ or more severe were significant risk factors for febrile UTI in spinal cord injury patients. Further prospective studies are necessary to define the full spectrum of possible risk factors for febrile UTI in these patients.

\section{DATA ARCHIVING}

There were no data to deposit.

\section{CONFLICT OF INTEREST}

The authors declare no conflict of interest.

1 Igawa Y, Wyndaele JJ, Nishizawa O. Catheterization: Possible complications and prevention and treatment. Int J Urol 2008; 15: 481-485.

2 Kakizaki H, Igawa Y, Gotou M, Takeda M, Hasegawa T, Koyanagi T et al. Clinical practice guideline for lower urinary tract dysfunction in patients with chronic spinal cord injury. J Jpn Med Soc Spinal Cord Lesion 2005; 16: 253-259.

3 Tanaka K, Hattori K, Hayashi H. Long-term follow up of intermittent catheterization by patient with spinal cord injury. J Jpn Med Soc Spinal Cord Lesion 2006; 19: 32-33.

4 Ozawa $\mathrm{H}$, Nishiyama $\mathrm{Y}$. Correlation between catheterization frequency and complication in spinal cord injured patients. J Jpn Med Soc Spinal Cord Lesion 2007; 20: 194-195.

5 Gerridzen RG, ThijssenAM, Dehoux E. Risk factors for upper tract deterioration inchronic spinal cord injury patients. J Urol 1992; 147: 416-418.

6 American Spinal Injury Association. International Standards for Neurological Classification of Spinal Cord Injury, Revised 2002. American Spinal Injury Association: Chicago, IL, USA, 2002.

7 Schurch B, Schmid DM, Karsenty G, Reitz A. Can neurologic examination predict type of detrusor sphincter-dyssynergia in patients with spinal cord injury? Urology 2005; 65: 243-246.

8 Takaba K, Shigemura K, Osawa K, Nomi M, Fujisawa M, Arakawa S. Emergence of extended-spectrum $\beta$-lactamase-producing Escherichia coli in catheter-associated urinary tract infection in neurogenic bladder patients. Am J Infect Control 2014; 42: e29-e31.

9 Beraldo PS, Neves EG, Alves CM, Khan P, Cirilo AC, Alencar MR. Pyrexia in hospitalised spinal cord injury patients. Paraplegia 1993; 31: 186-191.

10 Schaeflfer AJ, Schaeffer EM. Infections and inflammations. In: Wein AJ, Kavoussi LR, Novick AC, Partin AW, Peters CA (eds). Campbell-Walsh Urology, 10th edn. ElsevierSaunders: Philadelphia, PA, USA, 2011, pp 257-326.

11 Everaert K, Oostra C, Delanghe J, Vande Walle J, Van Laere M, Oosterlinck W. Diagnosis and localization of a complicated urinary tract infection in neurogenic bladder disease by tubular proteinuria and serum prostate specific antigen. Spinal Cord 1998; 36: 33-38.

12 Hooton TM, Bradley SF, Cardenas DD, Colgan R, Geerlings SE, Rice JC et al. Diagnosis, prevention, and treatment of catheter-associated urinary tract infection in adults: 2009 International Clinical Practice Guidelines from the Infectious Diseases Society of America. Clin Infect Dis 2010; 50: 625-663.

13 Nicolle LE. AMMI Canada Guidelines Committee. Complicated urinary tract infection in adults. Can J Infect Dis Med Microbiol 2005; 16: 349-360.

14 Kikkawa K, Mitsuda M, Yamasaki K, Uekado Y. Evaluation of urinary tract infection in patients treated with clean intermittent catheterization. Jpn J Environ Infect 2010; 25: 73-78.

15 Furuse A, Nagano K, Komaki S, Mochinaga H, Saitou M. Clinical analysis of childhood urinary tract infection due to extended-spectrum $\beta$-lactamase producing Escherichia coli and Klebsiella pneumoniae. J Pediatr Infect Dis Immunol 2014; 5: 403-406.

16 Quale JM, Landman D, Bradford PA, Visalli M, Ravishankar J, Flores C et al. Molecular epidemiology of a citywide outbreak of extended-spectrum -lactamase-producing Klebsiella pneumoniae infection. Clin Infect Dis 2002; 35: 834-841.

17 Kurtaran B, Candevir A, Tasova Y, Kibar F, Inal AS, Komur S et al. Antibiotic resistance in community-acquired urinary tract infections: prevalence and risk factors. Med Sci Monit 2010; 16: CR246-CR251.

18 Chong $\mathrm{Y}$, Shimoda S, Yakushiji $\mathrm{H}$, Ito $\mathrm{Y}$, Miyamoto $\mathrm{T}$, Kamimura $\mathrm{T}$ et al. Community spread of extended spectrum $\beta$-lactamase-producing Escherichia coli, Klebsiella pneumoniae and Proteus mirabilis: a long-term study in Japan. J Med Microbiol 2013; 62: 1038-1043.

19 Pena C, Pujol M, Ardanuy C, Ricart A, Pallares R, Liñares J et al. Epidemiology and successful control of a large outbreak due to Klebsiella pneumoniae producing extended-spectrum $\beta$-lactamases. Antimicrob Agents Chemother 1998; 42: $53-58$

20 Rahal JJ, Urban C, Horn D, Freeman K, Segal-Maurer S, Maurer J et al. Class restriction of cephalosporin use to control total cephalosporin resistance in nosocomial Klebsiella. JAMA 1998; 280: 1233-1237.

21 Sauerwein D. Urinary tract infection in patients with neurogenic bladder dysfunction. Int J Antimicrob Agents 2002; 19: 592-597.

22 Massa LM, Hoffman JM, Cardenas DD. Validity, accuracy and predictive value of urinary tract infection signs and symptoms in individuals with spinal cord injury on intermittent catheterization. J Spinal Cord Med 2009; 32: 568-573.

23 Jayawardena V, Midha M. Significance of bacteriuria in neurogenic bladder. J Spinal Cord Med 2004; 27: 102-105.

24 Cardenas DD, Hoffman JM, Kelly E, Mayo ME. Impact of a urinary tract infection educational program in persons with spinal cord injury. J Spinal Cord Med 2004; 27 : 47-54.

25 Cespedes RD, Gerboc JL. Other therapies for storage and emptying failure. In: Wein AJ, Kavoussi LR, Novick AC, Partin AW, Peters CA (eds). Campbell-Walsh Urology, 10th edn. Elsevier-Saunders: Philadelphia, CA, USA, 2011, pp 2186-2203.

26 Kovindha A, Na Chiang Mai W, Madersbacher H. Reused silicone catheter for clean intermittent catheterization (CIC): is it safe cord-injured (SCI) men? Spinal Cord 2004; 42: 638-642. 